\title{
Countercurrent Flow Limitations in a Pressurized Water Reactor
}

\author{
Deendarlianto, ${ }^{1}$ Thomas Höhne, ${ }^{2}$ and Michio Murase ${ }^{3}$ \\ ${ }^{1}$ Department of Mechanical \& Industrial Engineering, Faculty of Engineering, Gadjah Mada University, Jalan Grafika No. 2, \\ Yogyakarta 55281, Indonesia \\ ${ }^{2}$ Helmholtz-Zentrum Dresden-Rossendorf e.V., Institute of Fluid Dynamics, P.O. Box 510 119, 01314 Dresden, Germany \\ ${ }^{3}$ Institute of Nuclear Technology, Institute of Nuclear Safety System, Incorporated, 64 Sata, Mihama-cho, Mikata-gun, \\ Fukui 919-1205, Japan
}

Correspondence should be addressed to Thomas Höhne, t.hoehne@hzdr.de

Received 6 March 2012; Accepted 6 March 2012

Copyright () 2012 Deendarlianto et al. This is an open access article distributed under the Creative Commons Attribution License, which permits unrestricted use, distribution, and reproduction in any medium, provided the original work is properly cited.

Steam generators in a pressurized water reactor (PWR) nuclear power plant transfer heat from a primary coolant (pressurized water at about $15 \mathrm{MPa}$ ) to a secondary coolant (pressurized water/steam at about $7 \mathrm{MPa}$ ). The primary coolant water is heated in the core and passes through the steam generators, where it transfers heat to the secondary coolant water to generate steam. The steam then drives a turbine that turns an electric generator. Steam is condensed and returns to the steam generator as feedwater. Hot leg pipes connect the reactor pressure vessel (RPV) and the steam generator (SG) and consist of a combination of horizontal sections, single or multiple elbows, and inclined or vertical sections depending on the manufacturer of the reactor [1].

In the event of hypothetical accident scenarios in PWR, emergency strategies have to be mapped out, in order to guarantee the reliable removal of decay heat from the reactor core, also in case of component breakdown. One essential passive heat removal mechanism is the reflux cooling mode. This mode can appear for instance during a small break lossof-coolant accident (LOCA) or because of loss of residual heat removal (RHR) systems during midloop operation at plant outage after the reactor shutdown. Here, the accident at Three Mile Island (TMI) Unit 2 in 1979 has already indicated that the core heat removal mechanism during a smallbreak (SB) LOCA should be understood more clearly [2]. It includes a comprehensive study on the natural circulation cooling capability, such as loop seal clearing and countercurrent flow limitation (CCFL).

In the scenario of an LOCA, it is considered that the reactor will be depressurized and vaporization will take place, thereby creating steam in the PWR primary side. Should this lead to "reflux condensation," which may be a favourable event progression, the generated steam will flow to the steam generator through the hot leg. This steam will condense in the steam generator, and the condensate will flow back through the hot leg to the reactor, resulting in countercurrent steam/water flow. In some scenarios, the success of core cooling depends on the behaviour of this countercurrent flow.

In most cases of the CCFL experiments, the liquid flow rate was kept constant, and the gas flow rate was increased and decreased in small increments and decrements, respectively. For small gas flow rate, the liquid film flows countercurrently with the gas phase. During this condition, the pressure difference inside the test section slightly increases with the gas mass flow rate. This regime is defined as the stable countercurrent flow. As the gas flow rate is gradually increased, thus, there is the maximum gas flow rate in which the downflowing water mass flow rate is equal to the inlet liquid mass flow rate. This point is defined as the onset of flooding or CCFL. With further increasing of the gas mass flow rate, the downflowing liquid mass flow rate is close to zero. This point corresponds to the zero liquid penetration. In this situation, the cooling of the reactor core from the hot leg is impossible but may be continued by coolant drained through the cold leg to the downcomer. The region between the CCFL and zero liquid penetration is defined as partial delivery region. In turn, when the gas flow rate is decreased, a point is reached where a fully counter-current gas-liquid two-phase flow is established. This is known as the deflooding point.

Over several decades, a number of experimental and theoretical studies of countercurrent gas-liquid two-phase 
flow have been carried out to understand the fundamental aspect of the flooding mechanism and to prove practical knowledge for the safety design of nuclear reactors. They included experimental, scaling parameter development, analytical and computational modeling. Starting from the pioneering work of Wallis [3], extensive experimental data base has been accumulated dealing with a diverse array of conditions from these studies, leading to the development of phenomenological correlations and scaling parameters of the CCFL. Meanwhile, as we can see in the open literature some contradictory conclusions about flooding mechanisms, they were carried out under the same experimental conditions. Therefore, it is very difficult to obtain a general conclusion from the available data.

On the other hand, the nuclear thermal-hydraulic community is also facing today interesting challenges. These include the development and validation of new mathematical and computational tools that will be used for improved and more detailed analysis as well as new generations of reactors. For this purpose, the computational fluid dynamics (CFD) tool is considered to be able to simulate most of twophase flow configurations encountered in nuclear reactor power plants. The introducing of CFD method on the countercurrent flow in a model of PWR hot leg includes the investigation of CCFL mechanisms, heat transfer effects, flow patterns, hysteresis behaviour, and the extension of the obtained flow behaviour from small scale to fullreactor scale. However, the available literature regarding this important topic is rare.

It is expected by the community that the development of a general model closer to physics and including less empiricism in the CFD model should be considered $[4,5]$. Such models are an essential precondition for the application of CFD codes to the modeling of flow-related phenomena in nuclear facilities. Here, the validation of the developed local geometry-independent models for mass, momentum, heat transfer, and scalar transport should be conducted. The necessary validation is performed by comparing model results against measured data.

In view of the above, it has been decided to bring out this special issue. The available papers include the state of the art of CCFL experiment in a simple inclined or vertical pipe, scale down PWR hot leg model, CFD modeling, and development of new data base using image processing technique. Thus, this special issue provides the reader with useful information on the progress of the works regarding CCFL in a PWR, and also on open questions, requirements for further research, modeling, and experimental data.

Deendarlianto

Thomas Höhne

Michio Murase
[2] H. Y. Jeong, "Prediction of counter-current flow limitation at hot leg pipe during a small-break LOCA," Annals of Nuclear Energy, vol. 29, no. 5, pp. 571-583, 2002.

[3] G. B. Wallis, One-Dimensional Two-Phase Flow, McGraw Hill, New York, NY, USA, 1969.

[4] T. Höhne, Deendarlianto, and D. Lucas, "Numerical simulations of counter-current two-phase flow experiments in a PWR hot leg model using an interfacial area density model," International Journal of Heat and Fluid Flow, vol. 32, pp. 10471056, 2011.

[5] N. Minami, M. Murase, and A. Tomiyama, "Countercurrent gas-liquid flow in a PWR hot leg under reflux cooling (II) numerical simulation of 1/15-scale air-water tests," Journal of Nuclear Science and Technology, vol. 47, no. 2, pp. 149-155, 2010 .

\section{References}

[1] Deendarlianto, T. Höhne, D. Lucas, and K. Vierow, "Gasliquid countercurrent two-phase flow in a PWR hot leg: a comprehensive research review," Nuclear Engineering and Design, vol. 243, pp. 214-233, 2012. 

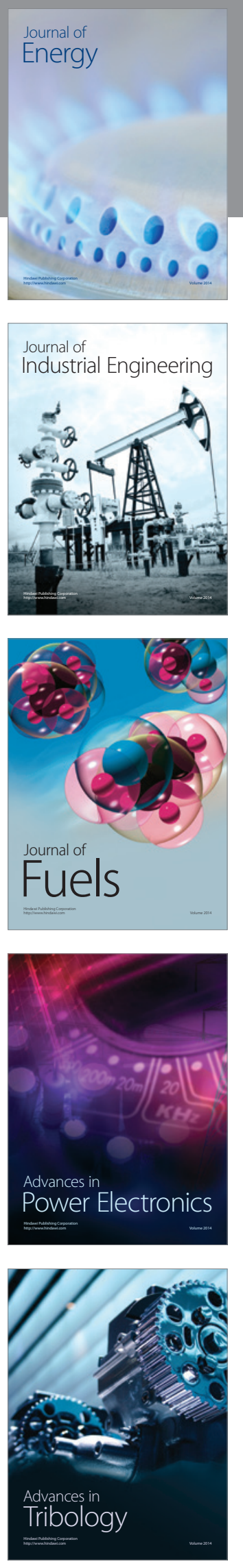
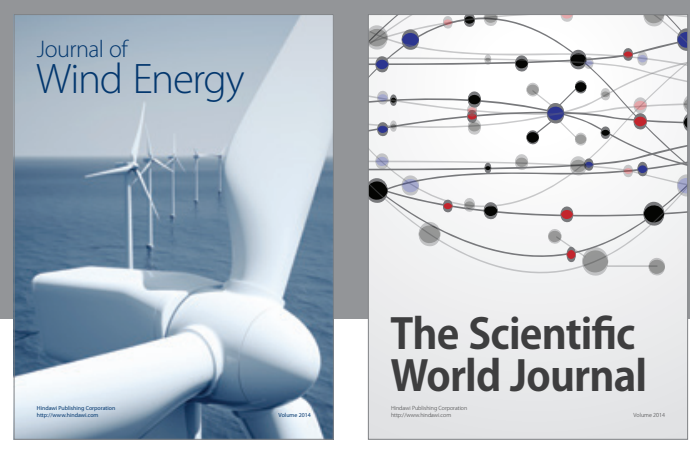

The Scientific World Journal

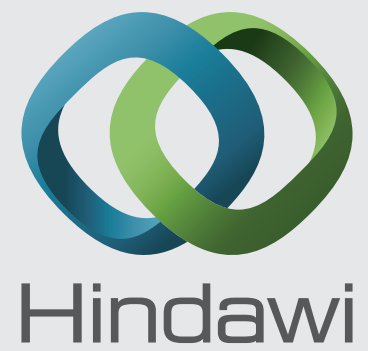

Submit your manuscripts at http://www.hindawi.com
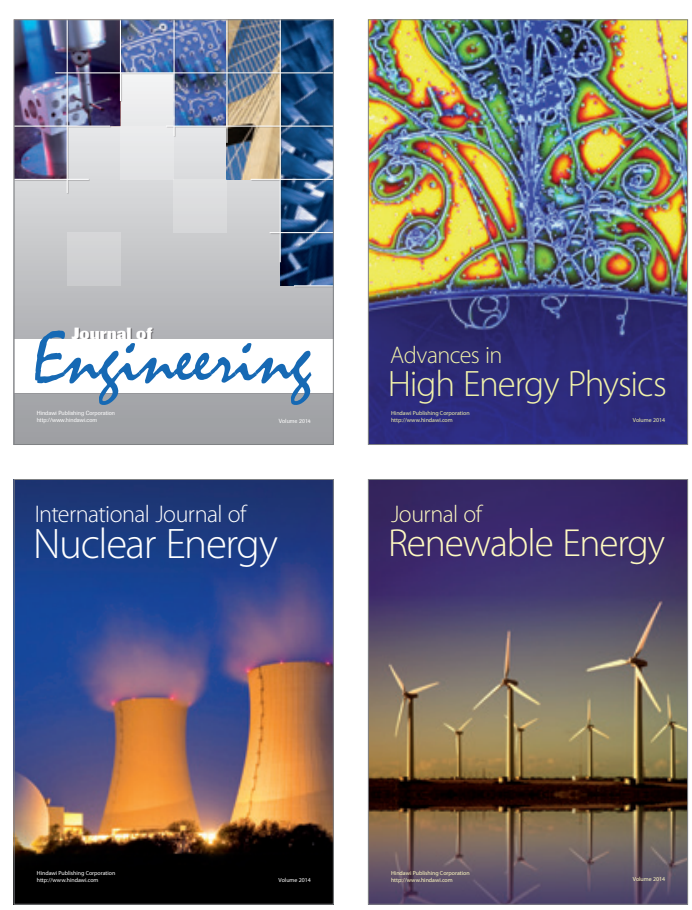

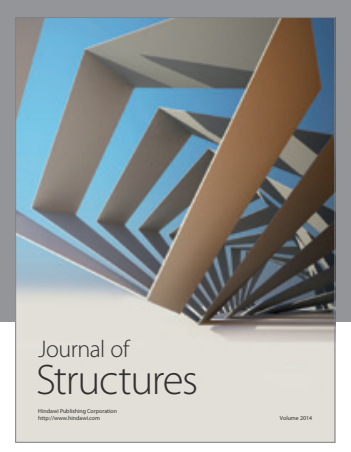

Rotating
Mechinery
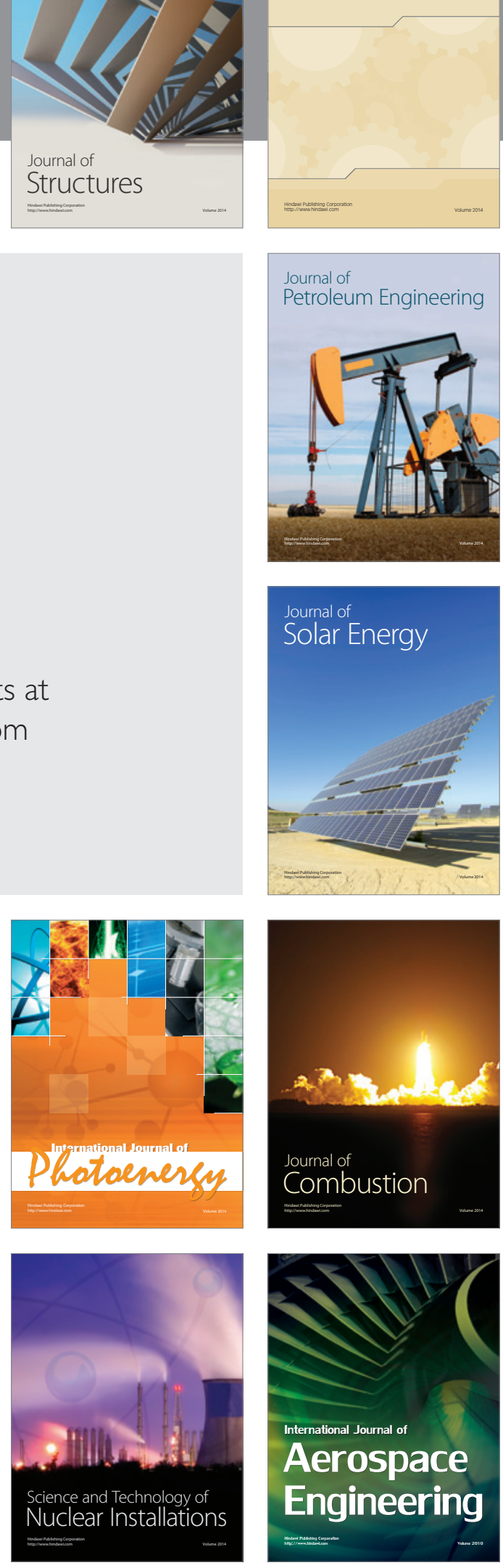